\title{
Simultaneous Integrated Boost using Conformal Radiation Therapy for Treatment of Cervical Cancer
}

\author{
Waqar M. Haque ${ }^{*}$, Eugene C. Endres², Sean Szeja, Sandra S. Hatch², Melissa M. Joyner ${ }^{2}$ \\ ${ }^{1}$ Greater Houston Physicians in Medicine Association, Houston, Texas \\ ${ }^{2}$ Department of Radiation Oncology, University of Texas Medical Branch, Galveston, Texas
}

"Corresponding Author: Waqar Haque, MD, Deparment of Radiation Oncology, Greater Houston Physicians in Medicine, Association Houston, TX 77030, Tel: 832-367-1655/ Fax: 832-201-0602; E-mail: WAQARH786@gmail.com

\begin{abstract}
Purpose: Chemoradiation is the treatment of choice for locally advanced cervical cancer. At our institution, we have been using forward planned conformal radiation therapy to treat patients to the whole pelvis with asimultaneous integrated boost (SIB) to the uterus and parametria. Our aim is to report the local control, disease free survival, overall survival, and toxicities of definitive chemoradiation with SIB for treatment of cervical cancer.

Methods: Medical records and treatment plans of patients with cervical cancer treated from 2009-2013 were reviewed using an IRB-approved database. The records of patients with cervical cancer treated with definitive chemoradiation and a three dimensional forward planned SIB were analyzed to determine local failure, distant failure, overall survival, and rate of toxicities.

Results: Twenty one patients were treated with definitive chemoradiation with a SIB. Median follow up time was 18.1 months. The 2-year LC rate was $95.2 \%$, the 2 -year DFS was $80.9 \%$, and there were no deaths, for an overall survival rate of $100 \%$. One patient experienced Grade 3 or higher acute toxicity, and two patients experienced Grade 3 or higher late toxicities.

Conclusions: This study demonstrates the feasibility and tolerability of SIB using forward planned conformal radiation therapy for the treatment of cervical cancer. This radiation technique can be used to deliver a higher dose to the area most at risk for recurrence in a shorter treatment time
\end{abstract}

Received Date: February 02, 2016

Accepted Date: April 21, 2016

Published Date: April 27, 2016

Citation: Haque, W.M., et al. Simultaneous Integrated Boost using Conformal Radiation Therapy for Treatment of Cervical Cancer. (2016) Intl J Cancer Oncol 3(1): 1- 7.

Keywords: Cervical cancer, Simultaneous integrated boost, Field-in-field 3D conformal radiation.

DOI: $10.15436 / 2377-0902.16 .802$

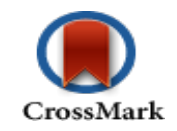

\section{Introduction}

Cervical cancer is the third most common gynecological cancer in the U.S., with an estimated 12,360 new cases diagnosed in $2014^{[1]}$. Despite excellent survival rates for localized disease, the estimated 5 year overall survival for patients with regional disease is only $57.4 \%^{[1]}$. Based on guidelines established by the National Comprehensive Cancer Network (NCCN) and the American Brachytherapy Society (ABS), the standard of care for patients with locally advanced cervical cancer is definitive chemotherapy and radiation therapy, including external beam radiation therapy (EBRT) and brachytherapy ${ }^{[2,3]}$. The target for EBRT includes gross disease, parametria, uterus, uterosacral ligaments, nodal volumes at risk, and sufficient vaginal margin from the gross disease. Improvements in technology have lead to the use of conformal radiation therapy and intensity modulated radiation therapy (IMRT) in an attempt to spare radiation toxicity to local organs, including the small bowel and the bone marrow ${ }^{[4-8]}$. Simultaneous integrated boost (SIB) is a radiation technique in which volumes of regional, suspected, and gross disease are treated simultaneously with a different dose per fraction dependent on the desired dose for the specific volume ${ }^{[9]}$. This technique was used for head and neck cancer and prostate cancer as early as 2000, and was first reported to be used in cervical cancer 2009 ${ }^{[9-11]}$. SIB allows a higher dose to be given to the tumor while shortening the overall treatment time, which may lead to an improvement in local control ${ }^{[12-14]}$. However, all current reports describing SIB for treatment of cervical cancer involve the use of inverse planned IMRT. Due to the steep dose gradients used in treatment with IMRT, movement of internal organs during treatment or between treatments can lead to geographical miss of the gross tumor volume or high dose administered to organs at risk. Given the variability of motion of internal organ motion, population- based planned tumor volume (PTV) margins would necessarily be large, possibly eliminating the benefits of IMRT ${ }^{[15]}$.

Copyrights: (C) 2016 Haque, W.M. This is an Open access article distributed under the terms of Creative Com- 
SIB using forward planned conformal radiation therapy for definitive chemoradiation for cervical cancer has not previously been explored. At our institution, in select patients, we have used a field-in-field technique to treat the whole pelvis with a SIB to the known areas of gross disease, cervix, and uterus. The purpose of this study is to describe the forward planned SIB technique and to report the clinical outcomes for patients with cervical cancer treated with forward planned SIB.

\section{Materials and Methods}

This analysis was approved by the institutional review board at the University of Texas Medical Branch and each of its affiliated hospitals. To determine the outcomes of patients with cervical cancer treated with definitive chemoradiation and $\mathrm{SIB}$, we reviewed the records of all patients with cervical cancer treated with radiation therapy at the University of Texas Medical Branch and affiliated hospitals from January 2009 to June 2013. We limited our analysis specifically to the 21 patients who received definitive chemoradiation with the SIB technique.

These patients' records and imaging were retrospectively reviewed to determine patient characteristics, tumor characteristics, clinical toxicities, and patient outcome. All patients underwent computed tomography (CT) Abdomen/Pelvis, Positron Emission Tomography (PET)/CT, and magnetic resonance imaging (MRI) for workup, and this information was used for treatment planning. Staging, however, was assigned based on clinical exam and plain films, per International Federation of Gynecology and Obstetrics (FIGO) guidelines. The primary endpoint was local control following chemoradiation. The response was determined by visual inspection, physical exam and palpation, or radiographic study including CT or MRI.

The whole pelvis was treated using a 3- dimensional (3D) conformal technique to a dose of 45 Gy in 25 fractions (1.8 Gy per fraction), with the exception of one patient, who was treated to a dose of 50.4 Gy in 28 fractions (1.8 Gy per fraction). For the anterior / posterior field, the superior border was the L4-L5 interspace, the inferior border was $3 \mathrm{~cm}$ inferior to gross disease or the obturator foramen, whichever was more inferior, and the lateral border was $2 \mathrm{~cm}$ lateral to the pelvic rim. The lateral fields shared the same superior and inferior borders as the A/P field, were bounded posteriorly by the sacrum, and was bordered anteriorly by the pubic symphysis. The SIB was utilized to treat the gross disease as visualized on PET/CT and MRI, uterus, and remnant of the cervix, with a $1.5 \mathrm{~cm}$ margin, named the CTV boost, upon which a $5 \mathrm{~mm}$ margin was applied to construct the PTV boost, to achieve a total dose of 50 Gy in 25 fractions (2 Gy per fraction) when the whole pelvis was treated to $45 \mathrm{~Gy}$. The one exception was in a patient treated to $50.4 \mathrm{~Gy}$ to the whole pelvis, where SIB achieved a dose of $54 \mathrm{~Gy}$ in 28 fractions (1.93 Gy per fraction) to the PTV boost. To accomplish this, initially the entire pelvic field was prescribed to be treated to $50 \mathrm{~Gy}$, after which field- in- field technique was performed to block the pelvic volume not enclosed by the PTV boost to treat this region to a dose of 45 Gy (Figures 1-3).

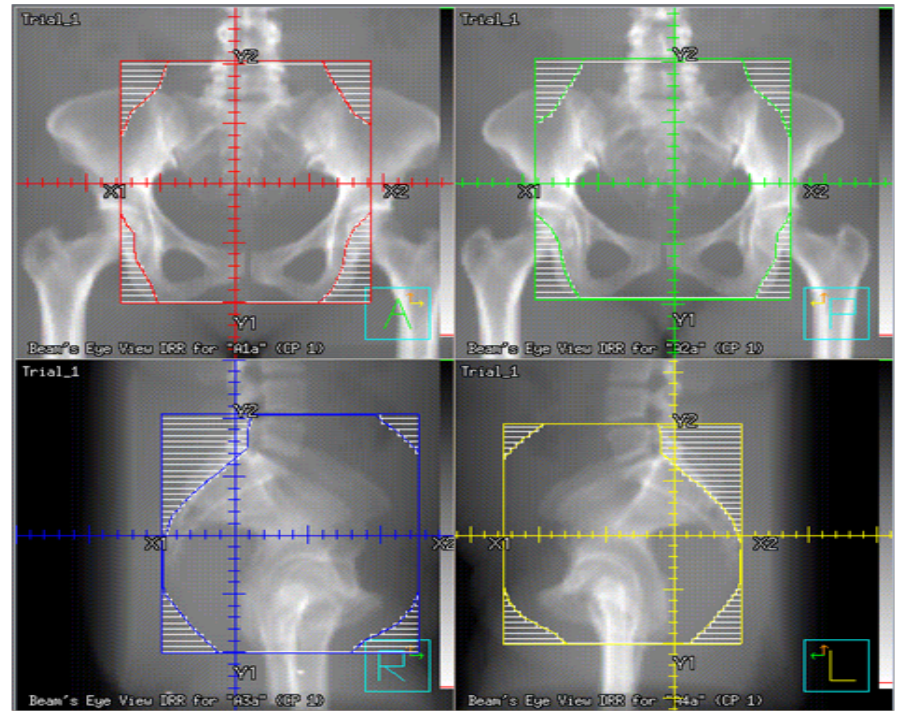

Figure 1: The anterior- posterior (AP), posterior- anterior (PA), and lateral fields used for treatment.

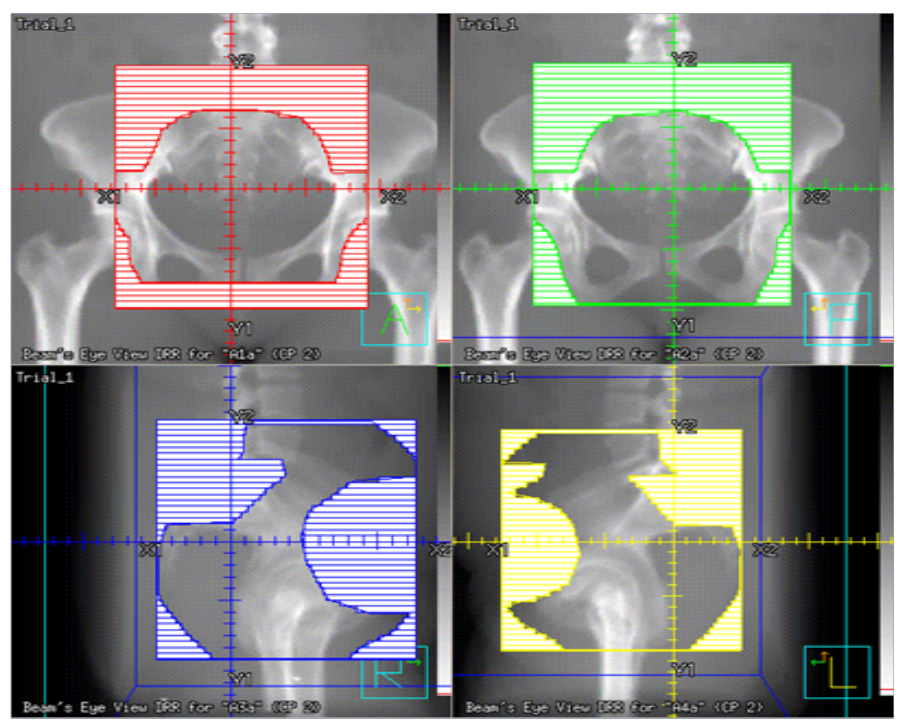

Figure 2: The field- in- field technique used to increase the dose to the region in the simultaneous integrated boost volume.

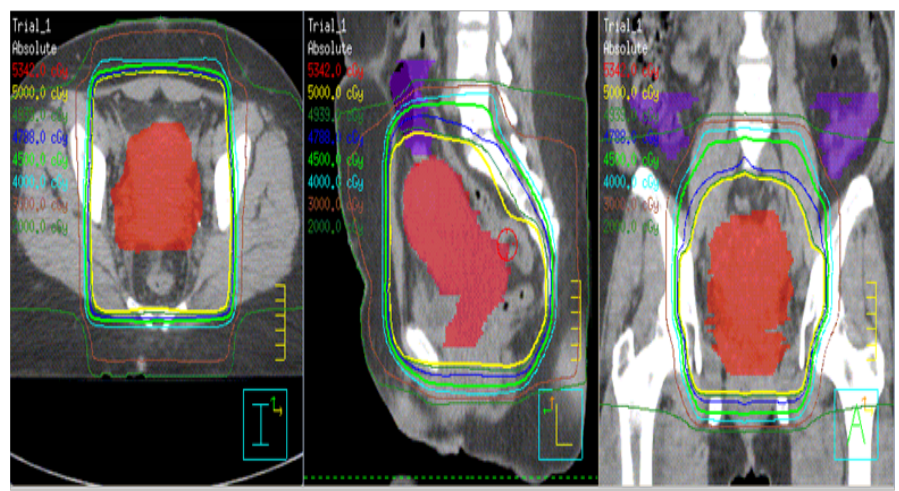

Figure 3: Resultant isodose lines for a patient treated with forward planned simultaneous integrate boost. 
High dose rate (HDR) intracavitary brachytherapy was used in each patient to assure a normalized equivalent dose (EQD2) of 80-90 Gy. HDR brachytherapy was started in the $4^{\text {th }}$ week of treatment, and each patient received 3 to 6 HDR insertions, 5 to 7.5 Gy per fraction. All patients were treated using tandem and ring with CT confirmation of placement. Relevant points as determined by ICRU 38 and the American Brachytherapy Society (ABS) guidelines included point $\mathrm{H}$, bladder, and rectal points ${ }^{[16]}$. In patients with nodal involvement, IMRT boost following completion of brachytherapy was used to take the nodal dose to 60-66 Gy. All patients were treated with concurrent weekly cisplatin $40 \mathrm{mg} / \mathrm{m} 2$, median 5 cycles given (range 2-6), with the determination of the number of cycles of chemotherapy administered determined by the treating gynecologic oncologist.

Data for age at treatment, stage, outcome, radiation dose, systemic therapy, and toxicity were retrospectively tabulated for all patients. Survival was defined as time from date of diagnosis to date of death or last follow-up (in months).

We performed Kaplan-Meier analyses to provide estimates of local control (LC) and disease free survival (DFS) for all patients. A local failure was defined as any evidence of tumor recurrence or progression within the pelvis discovered either on physical exam or on surveillance imaging by CT or PET scan. Data analysis was performed using SAS software version 9.3 (SAS Institute, Inc., Cary, NC). Toxicities were categorized by the Common Terminology Criteria for Adverse Events (CTCAE) version 4.0. All radiation therapy was delivered using a Varian linear accelerator with $6 \mathrm{MV}$ and $18 \mathrm{MV}$ photons (Varian Medical Systems, Palo Alto, California). Treatment planning was done using Pinnacle (Pinnacle Systems, Mountain View, California) or Eclipse Treatment Planning System (Varian Medical Systems, Palo Alto, California).

\section{Results}

Twenty one patients with cervical cancer were treated with radiation therapy at the University of Texas Medical Branch and affiliated hospitals between January 2009 - June 2013 and were treated with definitive chemoradiation with a SIB. Two $(9.5 \%)$ of these patients were American Joint Committee on Cancer (AJCC) stage IB1, six (28.6\%) were stage IIB, twelve $(57.1 \%)$ were stage IIIB, and one $(4.8 \%)$ was stage IVA. Four (19\%) of the patients had nodal disease discovered on imaging by PET / CT. Every patient included in the present analysis had squamous cell carcinoma. For further information regarding patient characteristics, please see Table 1.
Table 1: Patient characteristics and demographic information.

\begin{tabular}{|c|c|}
\hline Characteristic & $\mathbf{N}(\%)$ \\
\hline Median age & 52 \\
\hline \multicolumn{2}{|l|}{ Race or ethnic group } \\
\hline Hispanic & $9(42.9 \%)$ \\
\hline African American & $9(42.9 \%)$ \\
\hline White & $3(14.3 \%)$ \\
\hline \multicolumn{2}{|l|}{ AJCC Stage } \\
\hline IA & $0(0 \%)$ \\
\hline IB & $2(9.5 \%)$ \\
\hline IIA & $0(0 \%)$ \\
\hline IIB & $6(28.6 \%)$ \\
\hline IIIA & $0(0 \%)$ \\
\hline IIIB & $12(57.1 \%)$ \\
\hline IVA & $1(4.8 \%)$ \\
\hline IVB & $0(0 \%)$ \\
\hline \multicolumn{2}{|c|}{ External Beam radiation Dose } \\
\hline 45 Gy & $20(95.2 \%)$ \\
\hline 50.4 Gy & $1(4.8 \%)$ \\
\hline \multicolumn{2}{|c|}{ High Dose Rate Fractionation } \\
\hline $3 \mathrm{fx} \times 7.5 \mathrm{~Gy}$ & $7(33.3 \%)$ \\
\hline 4 fx x 7 Gy & $8(38.1 \%)$ \\
\hline $5 \mathrm{fx} \times 6 \mathrm{~Gy}$ & $5(23.8 \%)$ \\
\hline $6 \mathrm{fx} \times 5 \mathrm{Gx}$ & $1(4.8 \%)$ \\
\hline \multicolumn{2}{|c|}{ Cycles of chemotherapy } \\
\hline 2 cycles & $2(9.5 \%)$ \\
\hline 3 cycles & $4(19.1 \%)$ \\
\hline 4 cycles & $5(23.8 \%)$ \\
\hline 5 cycles & $9(42.9 \%)$ \\
\hline 6 cycles & $1(4.8 \%)$ \\
\hline
\end{tabular}

Among the patients with IB1 disease who received chemotherapy, one of these patients had evidence of PET positive external iliac and common iliac nodal disease, as a result of which our group recommended concurrent chemotherapy along with definitive radiation therapy. The second patient with IB1 disease who was treated with concurrent chemotherapy was found to have disease limited to $2.5 \mathrm{~cm}$ on clinical exam, but was found to have extension of PET avid disease in the lower uterine segment, suggesting the size of her tumor was significantly larger than the exophytic mass visualized on clinical exam, and as such was deemed to be an appropriate candidate for chemoradiation.

Twenty $(95.2 \%)$ of the patients were treated to the whole pelvis using a 3D conformal technique to a dose of $45 \mathrm{~Gy}$ in 25 fractions (1.8 Gy per fraction), with a SIB to the clinical gross disease to $50 \mathrm{~Gy}$ in 25 fractions ( 2 Gy per fraction). One $(4.8 \%)$ of the patients was treated to the whole pelvis to a dose of 50.4 Gy in 28 fractions (1.8 Gy per fraction) with a SIB boost to 54 Gy in 28 fractions (1.93 Gy per fraction). Four (19\%) of the patients received an additional IMRT boost to pathologic nodes found on PET imaging to an additional median dose of 13 Gy (10.8 Gy - 16.2 Gy). 
After a median follow up time of 18.1 months (range 366 months), two patients recurred locally, at four months and at twenty-nine months after completion of treatment, respectively. Three patients developed distant metastases. One patient failed in the lung at three months, one in the para-aortic nodes at four months, and one in the left supra clavicular node at 20 months, respectively. The 2-year LC rate was $95.2 \%$, the 2-year DFS was $80.9 \%$, and there were no deaths, for an overall survival rate of $100 \%$. Kaplan-Meier curves for LC and DFS are displayed in Figure 4.
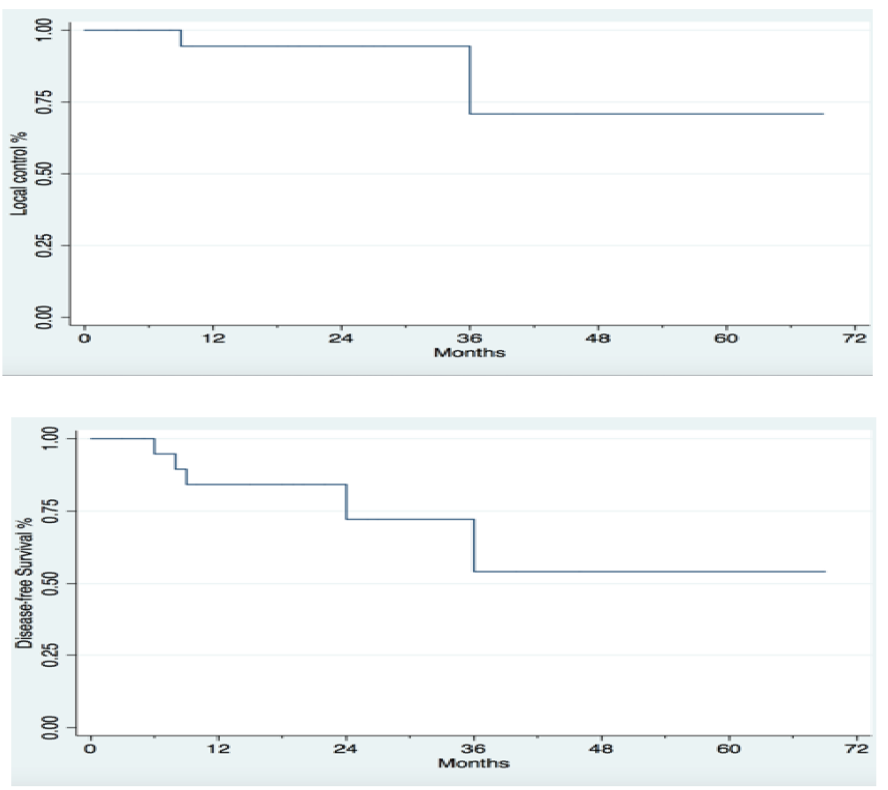

Figure 4: Kaplan-Meier estimates for local control and disease-free survival for patients with cervical cancer treated with simultaneous integrated boost.

Overall, radiation therapy was well tolerated in our patient population. Five $(23.8 \%)$ patients experienced no acute toxicities, twelve $(57.1 \%)$ patients experienced Grade 1 acute toxicities, three (14.3\%) patients experienced Grade 2 acute toxicities, and one (4.7\%) patient experienced Grade 3 acute toxicities in two distinct organ systems (Table 2). No Grade 4 or Grade 5 acute toxicities were observed.Sixteen (76.2\%) patients experienced no late toxicities, three (14.3\%) experienced Grade 1 late toxicities, and two (9.4\%) patients experienced Grade 3 late toxicities (Table 3 ).

Table 2: Acute toxicities.

\begin{tabular}{|l|c|c|c|c|c|}
\hline Toxicity & Grade 1 & Grade 2 & Grade 3 & Grade 4 & Grade 5 \\
\hline Skin & 4 & 1 & 0 & 0 & 0 \\
\hline $\begin{array}{l}\text { Bladder } \\
\text { abnormal- } \\
\text { ities }\end{array}$ & 3 & 0 & 0 & 0 & 0 \\
\hline Nausea & 1 & 2 & 1 & 0 & 0 \\
\hline $\begin{array}{l}\text { Bowel or } \\
\text { rectal ab- } \\
\text { normalities }\end{array}$ & 6 & 1 & 1 & 0 & 0 \\
\hline Fatigue & 4 & 0 & 0 & 0 & 0 \\
\hline
\end{tabular}

Table 3: Chronic toxicities.

\begin{tabular}{|l|c|c|c|c|c|}
\hline Toxicity & Grade 1 & Grade 2 & Grade 3 & Grade 4 & Grade 5 \\
\hline Skin & 0 & 0 & 0 & 0 & 0 \\
\hline $\begin{array}{l}\text { Bladder ab- } \\
\text { normalities }\end{array}$ & 2 & 0 & 1 & 0 & 0 \\
\hline Nausea & 0 & 0 & 0 & 0 & 0 \\
\hline $\begin{array}{l}\text { Bowel or } \\
\text { rectal abnor- } \\
\text { malities }\end{array}$ & 1 & 0 & 2 & 0 & 0 \\
\hline Fatigue & 0 & 0 & 0 & 0 & 0 \\
\hline
\end{tabular}

\section{Discussion}

The present study examined the outcomes of treating patients with cervical cancer with concurrent chemotherapy and EBRT using forward planned SIB using a field- in- field technique and HDR brachytherapy, followed by an IMRT boost if needed for PET avid lymph nodes. We demonstrated that EBRT using an SIB technique results in excellent local control, with 2-yr LC rate of $95.2 \%$, in a group of patients with predominantly locally advanced cervical cancer ( $>75 \%$ had FIGO stage IIB or higher disease). These local control rates were achieved with low toxicity rates, with $4.7 \%$ of patients experiencing grade 3 or higher acute toxicity, and $9.4 \%$ of patients experiencing grade 3 or higher late toxicity. This suggests that using EBRT with forward planned SIB is a safe technique that leads to excellent local control. This is to our knowledge the first report describing SIB using forward planned conventional treatment for definitive chemoradiation to treat cervical cancer.

Three groups have previously reported on the use of SIB with EBRT for definitive chemoradiation for treatment of cervical cancer, each of which utilized IMRT to achieve the SIB. The use of SIB in treatment of cervical cancer was first described by Vandecasteele et al. in $2009^{[11]}$. This was a review that described the planning procedure and feasibility of using Intensity Modulated Arc Therapy (IMAT) in six patients with unresectable cervical cancer in which the outcomes were not reported. In a subsequent prospective trial, the same group treated 30 patients with cervical cancer with IMAT and SIB and chemotherapy followed by hysterectomy ${ }^{[17]}$. There were no grades, 3 or higher early toxicities attributed to radiation therapy, and five $(16.6 \%)$ grade 3 or higher late toxicities. The 2-year local control rate was $96 \%$, and 2 -year distant control rate was $92 \%$. A German group described treatment of 40 patients with cervical cancer using helical tomotherapy with SIB and chemotherapy and HDR brachytherapy followed by curettage ${ }^{[18,19]}$. Acute Grade 3 diarrhea occurred in $2.5 \%$ of patients, acute Grade 3 hematologic toxicity occurred in $20 \%$ of patients, and $2 / 40$ patients had residual disease following curettage. Cihoric et al. described the outcomes of 10 patients with lymph node positive cervical cancer treated with IMRT and SIB followed by HDR brachytherapy ${ }^{[20]}$. At a median follow up time of 20 months, the local control rate was $90 \%, 10 \%$ of patients developed grade 3 acute toxicities, and $10 \%$ of patients developed grade 3 late toxicities. Patients treated in the present trial with a conformal forward planned SIB have similar rates of local control and acute and late toxicity, without the use of IMRT.

Another group evaluated the use of a SIB with chemoradiation in the neo adjuvant setting for locally advanced cervi- 
cal carcinoma for 32 patients in a phase I dose escalation trial ${ }^{[21]}$. These patients received concurrent cisplatin and 5FU, following which clinical responders underwent radical hysterectomy and lymphadenectomy. The rate of G3 or higher acute toxicities was up to $60 \%$ in the arm with the highest dose. No patients in this trial reported Grade 3 or higher late toxicities. With a median follow up time of 18 months, the LC was $68.7 \%$, DFS was $63.5 \%$, and OS was $92 \%$. It may be difficult to directly compare the toxicity rates of this trial and our present report due to the absence of brachytherapy use and inclusion of surgery. Nevertheless, the rate of Grade 3 or higher acute toxicities in both Group $2(25 \%)$ and Group $3(60 \%)$ were higher than the rate of Grade 3 or higher acute toxicities in the present trial $(4.7 \%)$, with the reported LC rate of $68.7 \%$ lower than the LC in the present trial of $95.2 \%$, suggesting the fractionation scheme used in the present trial plus HDR Brachytherapy may be superior in terms of both toxicity profile and local control.

Based on 5 randomized trials, there is evidence for a significant overall survival benefit for treatment of cervical cancer with the concurrent chemotherapy and radiation therapy when compared to radiotherapy alone ${ }^{[22]}$. The two largest trials that demonstrated improved outcomes with definitive treatment with concurrent cisplatin based chemoradiation over radiation therapy alone were the Radiation Therapy Oncology Group (RTOG) 90-01 and the Gynecologic Oncology Group (GOG) 120 (23-26). In RTOG 90-01, 403 patients with bulky IB to IVA cervical cancer were randomized to receive treatment with external beam radiation with or without with concurrent cisplatin and 5FU, with both arms receiving intracavitary brachytherapy (67\% of these patients had IIB-IVA disease). Loco regional failure (18\% vs. $34 \%)$, DFS (61\% vs. $36 \%)$, and OS $(67 \%$ vs. $41 \%$ ) all favored the chemoradiation arm. The rate of Grade 3 or higher acute toxicity was $45 \%$ in the chemoradiation arm, with the rate of non-hematologic acute toxicity being $11 \%$, and the rate of long term Grade 3 or higher toxicity was $13 \%{ }^{[23,24]}$. In GOG 120,526 patients with IIB, III, or IVA cervical cancer were randomized to receive one of three chemotherapy regimens: cisplatin alone; cisplatin, 5FU, and hydroxyurea; or hydroxyurea alone along with radiation therapy ${ }^{[25,26]}$. At ten years, the rates of progression free survival (46\%) and OS (53\%) favored the arms containing cisplatin ${ }^{[27]}$. The rate of grade 3 or higher long term toxicity was $4.7 \%$ for the cisplatin alone arm, $0.9 \%$ for the arm of patients receiving three drug treatment, and $2.1 \%$ for those received hydroxyurea alone. The rates of acute and late toxicity in the present study compare favorable to those reported in these historical controls, further suggesting that the SIB can be safely used without causing significant toxicity. Addtionally, rates of LC and OS in the present study compare favorably to the historical controls, demonstrating the efficacy of the SIB.

There are radiobiologic reasons to explain why treatment with SIB may potentially be more effective in treating patients with cervical cancer than conventionally fractionated radiation treatment followed by a cone down boost. Using SIB allows the treatment time to be decreased, limiting the effect of accelerated tumor repopulation ${ }^{[28]}$. Prolonging overall treatment time of patients with cervical cancer being treated with radiation therapy alone or with chemoradiation leads to worse cancer control outcomes ${ }^{[29-34]}$. Moreover, using the SIB treats the tumor to a slightly higher biologically effective dose (BED). Using the equation to calculate BED when taking into account solely the external beam radiation portion of treatment, $[\mathrm{BED}=\mathrm{nd}\{1+\mathrm{d} /$ $\left.(\alpha / \beta)\}-\ln 2\left(\mathrm{~T}-\mathrm{T}_{\mathrm{k}}\right) /\left(\alpha^{*} \mathrm{~T}_{\mathrm{p}}\right)\right]$ demonstrates that when using SIB to treat to 50 Gy in 25 fractions, the BED to the tumor is 56.92 Gy (assuming a $\alpha / \beta$ ratio of 10 for cervical cancer, $T_{k}$ of 19 days, $\mathrm{T}_{\mathrm{p}}$ of 4.5 days, and $\alpha$ of 0.3 / Gy), where as using conventional fractionation to treat to 45 Gy followed by a 5.4 Gy boost for a total of 28 daily fractions, the BED to the tumor is $54.95 \mathrm{~Gy}$. The combination of the decrease in treatment time along with the increase in BED may contribute to greater tumor kill and consequently superior oncologic outcomes.

The present study describes the use of SIB with forward planned conventional radiation therapy. Previous trials that described the use of SIB as part of definitive chemoradiation for management of cervical cancer used inverse planned IMRT. IMRT requires precise delineation of the target during and between treatments, which can be challenging in the case of the cervix. Studies have demonstrated that interfraction uterine motion can be up to $48 \mathrm{~mm}$, and interfraction cervical motion can be up to $19 \mathrm{~mm}^{[34]}$. Population based PTV margins may be larger than needed for some patients, which can cause unnecessary radiation treatment to organs at risk. Though individualized margins may be a potential solution, an adaptive strategy has yet to be clinically validated ${ }^{[15]}$. Therefore, due to positional uncertainty of the cervix which necessitates large PTV margins, the conformality typically achieved with IMRT may not be applicable in the case of cervical cancer. Additionally, conventional radiation is associated with a decreased cost of delivery and decreased planning time. This result is especially important given the increased emphasis on decreasing health care related costs while maintaining high quality treatment. Using 2015 Medicare reimbursement rates, a 25 fraction treatment for cervical cancer using IMRT would cost approximately $\$ 12700$, whereas a 25 fraction treatment regimen using $3 \mathrm{D}$ conformal treatment would cost approximately $\$ 4800$, with a resultant net savings of $\$ 7900$ per patient ${ }^{[35]}$. When extrapolated to the entire US population, treatment of all patients diagnosed with cervical cancer with 3D conformal treatment as opposed to IMRT would result in savings of $\$ 97,170,000$ annually. As the present study suggests, SIB using forward planned 3D conformal radiation achieves similar oncologic outcomes to SIB using IMRT while maintaining low toxicity, which may make it a more efficient, cost- effective treatment.

Our institutional data demonstrates that use of conventional 3D conformal radiation with forward planned SIB technique can achieve excellent local control with a reasonable toxicity profile. We did see a small number of Grade 3 acute and late toxicities. There was one patient who experienced who experienced Grade 3 acute nausea / vomiting which may have been partially due to chemotherapy, cisplatin. Two patients experienced Grade 3 or higher late toxicities, one of whom experienced Grade 3 rectal bleeding, successfully managed with hyperbaric oxygen therapy. This patient had a history or rheumatoid arthritis and was on Methotrexate, and was taking Methotrexate while receiving radiation treatment. Methotrexate is known to cause intestinal mucositis and gastrointestinal bleeding, and this may have contributed to the rectal bleeding experienced by this patient ${ }^{[36]}$. It is possible that the SIB technique should be avoided inpatients on agents that damage the gastrointestinal mucosa, as this may predispose patients to significant risk for severe toxicity. 
This study did have some weaknesses. Our cohort was a small, single-institution cohort, which may limit the generalizability of the findings. However, there was a good distribution of patients with different stages of cervical cancer within this cohort, which may make the results from this study generalizable to the general population. This was a retrospective study, which may lead to the possibility of underreporting of toxicities. Each patient was followed up closely by gynecologic oncologists and radiation oncologists specializing in treating gynecologic cancers, and as such we would expect any underreporting of toxicities to be minimal. Finally, this study had a limited follow up time, with a median follow up time of 18.1 months. Despite the limited follow up time, the excellent local control and disease free survival outcomes are significant, although longer follow up will be needed to determine the true effect of the SIB.

\section{Conclusion}

The present study described the technique and demonstrates the safety and efficacy of using SIB with forward planned $3 \mathrm{D}$ conformal radiation treatment for definitive chemoradiation for cervical cancer. This technique leads to excellent local control with minimal acute and long term toxicities. Oncologic outcomes are at least comparable to historical controls, and may be superior, due to decreased treatment time and a higher BED to the tumor. Longer follow up time will be needed to confirm the outcomes with treatment with SIB using 3D conformal radiation treatment.

Conflict of Interest: There are no conflicts to disclose.

\section{References}

1. SEER Stat Fact Sheets: Testis Cancer. Accessed April 16, 2015.

2. National Comprehensive Cancer Network. Cervical Cancer V2.2015. Accessed December 24, 2014.

3. Viswanathan, A.N., Thomadsen, B. American Brachytherapy Society consensus guidelines for locally advanced carcinoma of the cerivx. Part I: General Principles. (2012) Brachytherapy 11(1): 33-46.

4. Mavroidis, P., Lind, B.K., Van Dijk, B., et al. Comparison of conformal radiatoin therapy techniques within the dynamic radiotherapy project 'Dynarad.' (2000) Phys med Biol: 45 (9): 2459-81.

5. Roeske, J.C., Lujan, A., Rotmensch, J., et al. Intensity-modulated whole pelvic radiation therapy in patients with gynecologic malignancies. (2000) Int J Radiat Oncol Biol Phys 48(5): $1613-$ 21.

6. Portelance, L, Chao, K.S., Grigsby, P.W., et al. Intensity-modulated radiation therapy (IMRT) reduces small bowel, rectum, and bladder doses in patients with cervical cancer receiving pelvic and para-aortic irradiation. (2001) Int J Radiat Oncol Biol Phys 51(1): 261-66.

7. Mundt, A.J., Lujan, A.E., Rotmensch, J., et al. Intensity-modulated whole pelvic Radiotherapy in women with gynecologic malignancies. (2002) Int J Radiat Oncol Biol Phys 52(5): 1330 37.

8. Heron, D.E., Gerszten, K., Selvaraj, R.N., et al. Conventional $3 \mathrm{D}$ conformal versus intensity-modulated radiotherapy for ad- juvant treatment of gynecologic malignancies: A comparative dosimetric study of dose-volume histograms. (2003) Gynecol Oncol 91(1): 39-45.

9. Wu, Q., Manning, M., Schmidt-Ullrich, R., et al. The potential for sparing of parotids and escalation of biologically effective dose with intensity-modulated radiation treatments of head and neck cancers: a treatment design study. (2000) Int J Radiat Oncol Biol Phys 46(1): 195-205.

10. Bos, L.J., Damen, E.M., de Boer, R.W., et al. Reduction of rectal dose by integration of the boost in the large-field treatment plan for prostate irradiation. (2002) Int J Radiat Oncol Biol Phys 52(1): 254-65.

11. Vandecasteele, K., De Neve, W., De Gersem, W., et al. Intensity-modulated arc therapy with simultaneous integrated boost in the treatment of primary irresectablecervical cancer. Treatment planning, quality control, and clinical implementation. (2009) Strahlenther Onkol 185(12): 799-807.

12. Choy, D., Wong L.C., Sham, J., et al. Dose-tumor response of carcinoma of cervix: an analysis of 594 patients treated by radiotherapy. (1993) Gynecol Oncol 49(3): 311-17.

13. Lanciano, R.M., Martz, K., Coia, L,R., et al. Tumor and treatment factors improving outcome in stage III-B cervix cancer. (1991) Int J Radiat Oncol Biol Phys 20(1): 95-100.

14. Niibe, Y., Hayakawa, K., Kanai, T., et al. Optimal dose for stage IIIB adenocarcinoma of the uterine cervix on the basis of biological effective dose. (2006) Eur J Gynaecol Oncol 27(1): 47-9.

15. Jadon, R., Pembroke, C.A., Hanna, C.L., et al. A systematic review of organ motion and image-guided strategies in external beam radiotherapy for cervical cancer. (2014) Clin Oncol (R Coll Radiol) 26(4): 185-96.

16. Nag, S., Erickson, B., Thomadsen, B., et al. The American Brachytherapy Society recommendations for high-dose-rate brachytherapy for carcinoma of the cervix. (2000) Int J Radiat Oncol Biol Phys 48(1): 201-11.

17. Vandecasteele, K., Makar, A., Van den Broecke, R., et al. Intensity-modulated arc therapy with cisplatin as neo-adjuvant treatment for primary irresectable cervical cancer. Toxicity, tumour response and outcome. (2012) Strahlenther Onkol 188(7): 576-81.

18. Marnitz, S., Stromberger, C., Kawgan-Kagan, M., et al. Helical tomotherapy in cervical cancer patients: simultaneous integrated boost concept:technique and acute toxicity. (2010) Strahlenther Onkol 186(10): 572-79.

19. Marnitz, S., Köhler, C., Burova, E., et al. Helical tomotherapy with simultaneous integrated boost after laparoscopic staging in patients with cervical cancer: analysis of feasibility and early toxicity. (2012) Int J Radiat Oncol Biol Phys 82(2): e137-43.

20. Cihoric, N., Tapia, C., Krüger, K., et al. IMRT with 18 FDGPET/ CT based simultaneous integrated boost for treatment of nodal positive cervical cancer. (2014) Radiat Oncol 9(83): 1-8.

21. Macchia, G., Ferrandina, G., Deodato, F., et al. Concomitant boost dose escalation plus large-field preoperative chemoradiation in locally advanced carcinoma of the uterine cervix: results of a phase I study (LARA-CC-1). (2010) Gynecol Oncol 118(2): 128-33.

22. Thomas, G.M. Improved treatment for cervical cancer- concurrent chemotherapy and radiotherapy. (1999) N Engl J Med 340(15): 1198-200.

23. Eifel, P.J., Winter, K., Morris, M., et al. Pelvic irradiation 
with concurrent chemotherapy versus pelvic and para-aortic irradiation for high-risk cervical cancer: an update of radiation therapy oncology group trial (RTOG) 90-01. (2004) J Clin Oncol 22(5): 872-80.

24. Morris, M., Eifel, P.J., Lu, J., et al. Pelvic radiation with concurrent chemotherapy compared with pelvic and para-aortic radiation for high-riskcervical cancer. (1999) N Engl J Med 340(15): 1137-43.

25. Rose, P.G., Bundy, B.N., Watkins, E.B., et al. Concurrent cisplatin-based radiotherapy and chemotherapy for locally advanced cervical cancer. (1999) N Engl J Med 340(15): 1144-53. 26. Rose, P.G., Ali, S., Watkins, E., et al. Long-term follow-up of a randomized trial comparing concurrent single agent cisplatin, cisplatin-based combination chemotherapy, or hydroxyurea during pelvic irradiation for locally advanced cervical cancer: a Gynecologic Oncology Group Study. (2007) J Clin Oncol 25(19): 2804-10.

27. Keys, H.M., Bundy, B.N., Stehman, F.B., et al. Cisplatin, radiation, and adjuvant hysterectomy compared with radiation and adjuvant hysterectomy for bulky stage IB cervical carcinoma. (1999) New Engl J Med 340(15): 1154-1161.

28. Withers, H., Taylor, J., Maciejewski, B., et al. The hazard of accelerated tumor clonogen repopulation during radiotherapy. (1988) Acta Oncol 27(2): 131-146.

29. Fyles, A., Keane, T.J., Barton, M., et al. The effect of treatment duration in the local control of cervix cancer. (1992) Radiother Oncol 25(4): 273-279.
30. Perez, C.A., Grigsby, P.W., Castro-Vita, H., et al. Carcinoma of the uterine cervix. I. Impact of prolongation of overall treatment time and timing of brachytherapy on outcome of radiation therapy. (1995) Int J Radiat Oncol Biol Phys 32(5): 1275-88.

31. Petereit, D.G., Sarkaria, J.N., Chappell, R., et al. The adverse effect of treatment prolongation in cervical carcinoma. (1995) Int J Radiat Oncol Biol Phys 32(5): 1301-1307.

32. Chen, S.W., Liang, J.A., Yang, S.N., et al. The adverse effect of treatment prolongation in cervical cancer by high-dose-rate intracavitary brachytherapy. (2003) Radiother Oncol 67(1): 6976.

33. Song, S., Rudra, S., Hasselle, M.D., et al. The effect of treatment time in locally advanced cervical cancer in the era of concurrent chemoradiotherapy. (2013) Cancer 119(2): 325-31.

34. Krebs, L., Maillard, S., Gaillot-Petit N., et al. Total radiation dose and overall treatment time are predictive for tumor sterilization in cervical carcinoma treated with chemoradiation and pulsed-dose-rate brachytherapy. (2015) Brachytherapy 14(1): $16-22$.

35. Taylor, A., Powell, M.E. An assessment of interfractional uterine and cervical motion: implications for radiotherapy target volume definition in gynaecological cancer. (2008) Radiother Oncol 88(2): 250-257

36. Find-a-code. Accessed October 21, 2015.

37. Tsukada, T., Nakano, T., Miyata, T., et al. Life- threatening gastrointestinal mucosal necrosis during Methotrexate treatment for Rheumatoid Arthritis. (2013) Case Rep Gastroenterol 7(3): 470-475.
Journal ISSN: 2377-0902

E-mail: cancerandoncology@ommegaonline.com Website: www.ommegaonline.org 